\title{
石墨烯量子点功能化金纳米粒子的制备及作为过氧化物模拟酶用于 葡萄糖检测
}

\author{
郭颖* 李午戊郑敏燕黄怡 \\ (咸阳师范学院化学与化工学院 咸阳 712000)
}

\begin{abstract}
摘要 基于石墨烯量子点(GQD)的还原性，在无需外加保护剂的条件下，采用一步法成功制备了稳定性高、分散性好的 石墨烯量子点功能化的金纳米粒子(GQD@AuNPs). 所制备的 GQD@AuNPs 有优良的过氧化物模拟酶催化活性, 能够 有效地催化 $\mathrm{H}_{2} \mathrm{O}_{2}$ 氧化 3,3',5,5'-四甲基联苯胺(TMB)产生显色反应. 以 $\mathrm{TMB}$ 为模型底物, 研究了催化条件(温度和 $\mathrm{pH}$ ) 对催化活性的影响. 电子自旋共振光谱(ESR)结果表明, GQD@AuNPs 与辣根过氧化物酶(HRP)类似, 能有效地催化 $\mathrm{H}_{2} \mathrm{O}_{2}$ 分解成羟基自由基( $\mathrm{OH}$ ). 基于此, 结合葡萄糖在匍萄糖氧化酶(GOx)作用下产生 $\mathrm{H}_{2} \mathrm{O}_{2}$ 的原理，建立了可视化检测 血清中葡萄糖含量的简便方法. 在优化条件下, 本方法的检测范围为 $2.0 \times 10^{-6} \sim 4.0 \times 10^{-5} \mathrm{~mol} \cdot \mathrm{L}^{-1}$, 检出限为 $3.0 \times 10^{-7}$ $\mathrm{mol} \cdot \mathrm{L}^{-1}$, 并对实际样品进行测定, 测定结果与临床结果一致.
\end{abstract}

关键词 石墨烯量子点; 纳米金; 过氧化物模拟酶; 葡萄糖

\section{Facile Preparation of Graphene Dots Functionalized Au Nanoparticles and Their Application as Peroxidase Mimetics in Glucose Detection}

\author{
Guo, Ying* \\ Li, Wuwu \\ Zheng, Minyan \\ Huang, Yi \\ (College of Chemistry \& Chemical Engineering, Xianyang Normal University, Xianyang 712000)
}

\begin{abstract}
Based on the reducing property of graphene dots, we developed a facile strategy to synthesize well-stable graphene dots functionalized Au nanoparticles (denoted as GQD@AuNP) by one-step process at low temperature without added protecting agent. The prepared GQD@AuNP were characterized by transmission electron microscopy (TEM), X-ray photoelectron spectroscopy (XPS) and UV-vis absorption spectra. It was found that the as-prepared GQD@AuNP are spherical and monodisperse with an average diameter of $c a .10 \mathrm{~nm}$. Graphene dots not only act as a mild reductant to reduce $\mathrm{HAuCl}_{4}, \mathrm{but}$ also as a capping agent to endow the GQD@AuNP with good stability in aqueous solvent and monodispersity and restrains the $\mathrm{Au}$ crystal growth, making the particles have narrow size distribution. Furthermore, the as-prepared GQD@AuNP showed an excellent intrinsic peroxidese-like activity, which could catalyze oxidization of 3,3',5,5'-tetramethylbiphenyl (TMB) by $\mathrm{H}_{2} \mathrm{O}_{2}$ to produce a colour variation. Using TMB as substrate, we systematically studied the effect of a series of conditions, such as temperature and $\mathrm{pH}$, on the catalytic activity of the as-prepared GQD@AuNP. Results of electron paramagnetic resonance (ESR) suggest that the catalyse-mimic activity of the GQD@AuNP like HRP and effectively catalyzed the decomposition of $\mathrm{H}_{2} \mathrm{O}_{2}$ into ${ }^{\circ} \mathrm{OH}$ radicals. On this basis, a highly sensitive and rapid colorimetric and visualization method was developed for glucose in blood samples, when combined with glucose oxidase (GOx). Under optimum conditions, the proposed method allowed the detection of glucose in the range of $2.0 \times 10^{-6}$ to $4.0 \times 10^{-5} \mathrm{~mol} \cdot \mathrm{L}^{-1}$ with detectable glucose as low as $3.0 \times 10^{-7} \mathrm{~mol} \cdot \mathrm{L}^{-1}$. Furthermore, another three sugars existing in the human serum, including maltose, fructose and lactose were detected by this method, which indicated the little disturbance by maltose and fructose, while no remarkable signals were observed for the lactose. This proposed method has been successfully applied to detect glucose in serum samples with good accuracy and precision.
\end{abstract}

Keywords graphene dots; Au nanoparticles; peroxidase mimetics; glucose

\section{1 引言}

金纳米粒子因具有比表面积大、表面反应活性高、 独特的催化性能等优良特性, 已成为一个相当重要的纳 米材料 ${ }^{[1 \sim 3]}$. 近年来, 纳米金在气相和液相氧化还原反
应体系中的催化研究成为一个迅速发展的领域 ${ }^{[4 ~ 6]}$. 其 催化性能与制备方法、粒子大小及表面修饰剂等因素密 切相关 ${ }^{[7 \sim 9]}$. 通过简单方法制备出单分散性好、催化性 能高的纳米金颗粒成为研究者追求的目标. 目前，纳米

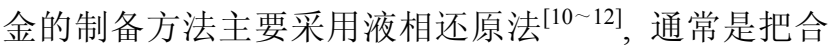

* E-mail: guoying207@126.com

Received February 26, 2014; published April 25, 2014.

Project supported by the National Natural Science Foundation of China (No. 21275096) and the Scientific Research Plan Projects of Education Department of Shaanxi Province (No. 2010JK898).

项目受国家自然科学基金(No. 21275096)和陕西省教育厅科学研究计划(No. 2010JK898)资助. 
适的还原剂如柠檬酸钠、硼氢化物等加入到反应液中, 通过还原 $\mathrm{Au}^{3+}\left(\mathrm{HAuCl}_{4}\right)$ 得到 $\mathrm{Au}^{0}$ 纳米金. 在这些方法中, 为避免纳米金颗粒的团聚, 通常在制备过程中需要添加 高分子表面活性剂、稳定剂(保护剂), 这些保护剂吸附 在粒子表面，通过静电或空间位阻的作用避免了粒子间 的直接接触, 使胶体粒子能稳定的存在于溶液中 ${ }^{[13,14]}$. 然而表面活性剂、稳定剂通常导电性差, 对催化剂的影 响很大, 有些会导致纳米金活性降低甚至丧失催化活 性, 因此寻找新型的导电性强的保护剂对于制备好的催 化剂尤为重要.

GQD, 作为碳材料家族的新型成员, 除了具有较好 的导电性能 ${ }^{[15,16]}$ 外还因量子限制效应和边界效应而展 现出一系列新的特性, 因此引起了人们的广泛关

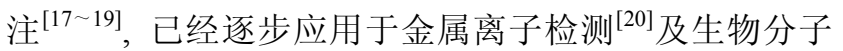
的检测 ${ }^{[21,22]}$. 然而, 目前大多数研宄者都关注 GQD 的
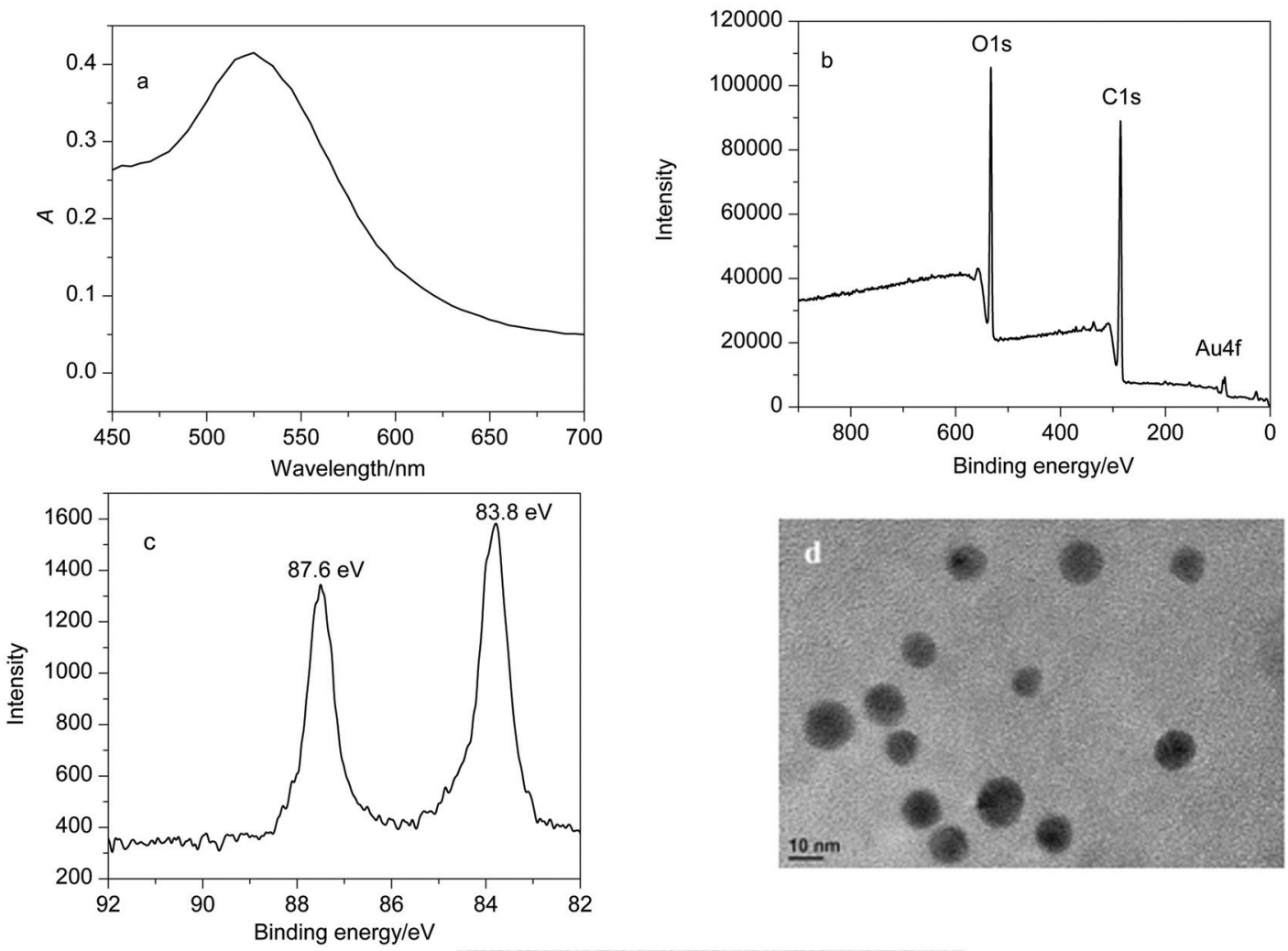

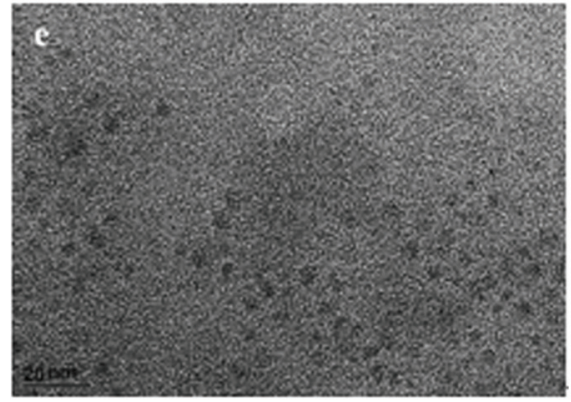

图 1 (a) GQD@AuNPs 的紫外-可见吸收光谱; (b) GQD@AuNPs 的 XPS 全扫描; (c) Au 的 XPS 谱图; (d) GQD@AuNPs 的 TEM 照片(标尺 10 nm); (e) GQD 的 TEM 照片(标尺 $20 \mathrm{~nm}$ )

Figure 1 (a) UV-vis spectra of GQD@AuNPs; (b) XPS spectra of GQD@AuNPs; (c) High-resolution Au4f peaks of GQD@AuNPs; (d) TEM image of GQD@AuNPs (bar: 10 nm); (e) TEM image of GQD (bar: 20 nm) 
共振吸收峰 ${ }^{[23]}$. 这一结果表明金纳米粒子的生成, 而且 粒径较小. 图 $1 b$ 是一个典型的 GQD@AuNPs 的 X 射线 光电子能谱(XPS)全图分析, 由图可知, 除了 $\mathrm{Au} 4 \mathrm{f}$ 峰 (ca. $84 \mathrm{eV})$ 外, 还出现 $\mathrm{C} 1 \mathrm{~s}$ 峰 $(c a .284 \mathrm{eV})$ 和 $\mathrm{O} 1 \mathrm{~s}$ 峰 $(c a$. $532 \mathrm{eV}$ ), 证明我们成功的制备了 GQD@AuNPs. 在 $\mathrm{Au} 4 \mathrm{f}$ 的高分辨谱线中(图 1c), 其 $\mathrm{Au} 4 \mathrm{f}_{7 / 2}$ 和 $\mathrm{Au} 4 \mathrm{f}_{5 / 2}$ 的电 子结合能分别对应于 83.8 和 $87.6 \mathrm{eV}$, 表明此时纳米金 是处于零价金属状态 ${ }^{[24]}$. 图 $1 \mathrm{~d}, 1 \mathrm{e}$ 分别为所制备的 GQD@AuNPs 和 GQD 的透射电子显微镜(TEM)图, 可 以看出制备的 GQD@AuNPs 分布比较均匀, 基本上为 椭球形纳米颗粒, 粒径分布在 $10 \mathrm{~nm}$ 左右. GQD 平均粒 径约为 $3.5 \mathrm{~nm}$.

\subsection{GQD 的作用}

本文是通过 GQD 与金属离子之间直接发生氧化还 原反应，制备了GQD@AuNPs. 用 XPS 对 GQD 在反应 前后进行了研究, 见图 2. C1s 谱图可拟合 4 个峰, 其中 石墨化碳 $(\mathrm{C}-\mathrm{C})$ 和酚碳或醚碳 $(\mathrm{C}-\mathrm{O})$ 含量在反应后发 生了变化, 相比于原 GQD, 在形成 GQD@AuNPs 后, 石 墨化碳含量明显增加, 而酚碳或醚碳 $(\mathrm{C}-\mathrm{O})$ 的含量明显 降低. 发生这种现象的解释是: 由于 GQD 表面的含氧 官能团将 $\mathrm{Au}^{3+}$ 还原成 $\mathrm{AuNPs}{ }^{[25,26]}$.
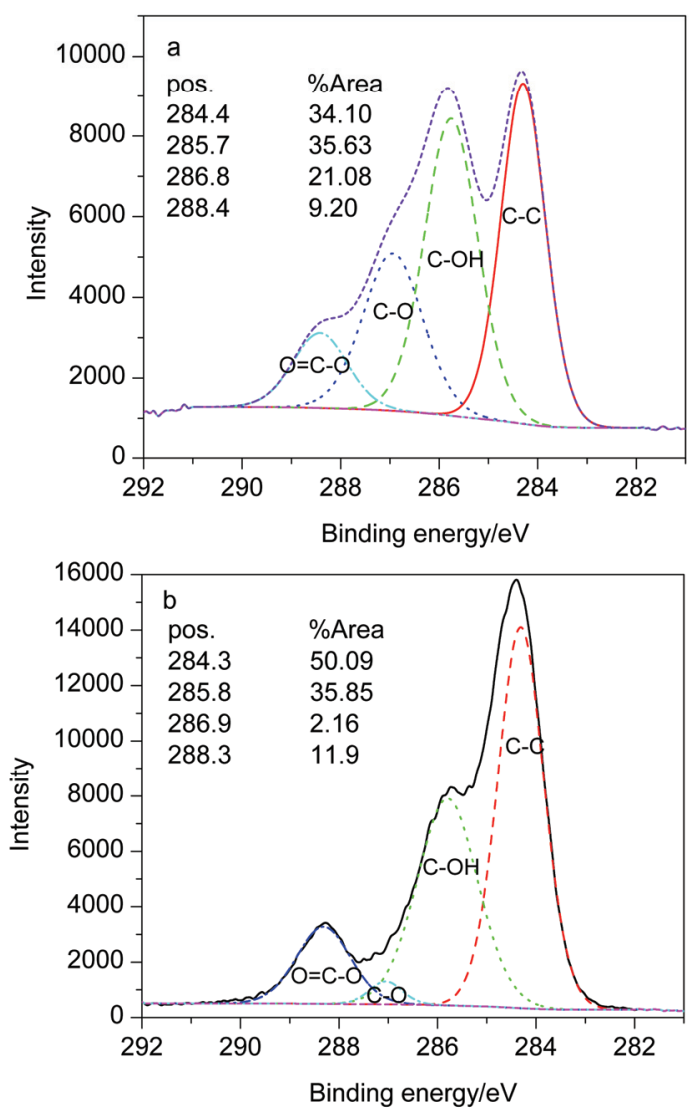

图 2 GQD (a) 和 GQD@AuNPs (b) 的 C1s XPS 谱图

Figure 2 XPS spectra of $\mathrm{C} 1 \mathrm{~s}$ on (a) GQD and (b) GQD@AuNPs

同时, 由于 GQD 表面含有羟基, 可以通过氢键作
用连接到 AuNPs 表面 ${ }^{[9]}$, 因此 GQD 的存在抑制了金纳 米颗粒自身的团聚，使其在水中呈良好的分散性，在空 气中静置三个月之后的纳米颗粒没有发生颜色变化或 团聚。

\subsection{GQD@AuNPs 的过氧化物模拟酶特性}

以过氧化物酶底物 $\mathrm{TMB}$ 为显色底物, $\mathrm{H}_{2} \mathrm{O}_{2}$ 为氧化 底物, GQD@AuNPs 作为催化剂, 考察该体系的显色反 应, 评价其过氧化物模拟酶的催化特性. 实验表明, $\mathrm{H}_{2} \mathrm{O}_{2}$ 不能单独氧化 TMB 显色, 而在 $0.20 \mathrm{~mol} \bullet \mathrm{L}^{-1}$ $\mathrm{HAc}-\mathrm{NaAc}$ 缓冲溶液中 $(\mathrm{pH} 3.50), \mathrm{GQD} @ A u N P s$ 能够催 化 $\mathrm{H}_{2} \mathrm{O}_{2}$ 氧化 $\mathrm{TMB}$ 产生经典的显色反应, 其氧化产物 ox-TMB 显蓝色, 在 $652 \mathrm{~nm}$ 处有一特征吸收峰. GQD@AuNPs 催化 $\mathrm{H}_{2} \mathrm{O}_{2}$ 氧化 TMB 的吸光度远远大于 单独 $\mathrm{H}_{2} \mathrm{O}_{2}$ 作用于 $\mathrm{TMB}$ 的吸光度 (图 3). 此外在 GQD@AuNPs 与 $\mathrm{H}_{2} \mathrm{O}_{2}$ 和 TMB 的显色溶液中加入 2.0 $\mathrm{mol} \cdot \mathrm{L}^{-1} \mathrm{H}_{2} \mathrm{SO}_{4}$ 后, 溶液颜色由蓝色迅速变为黄色, 显 色反应停止，与 HRP 催化 TMB 与 $\mathrm{H}_{2} \mathrm{O}_{2}$ 显色反应一样. 这些结果表明, GQD@AuNPs 具有与 HRP 类似的过氧化 物酶催化活性.

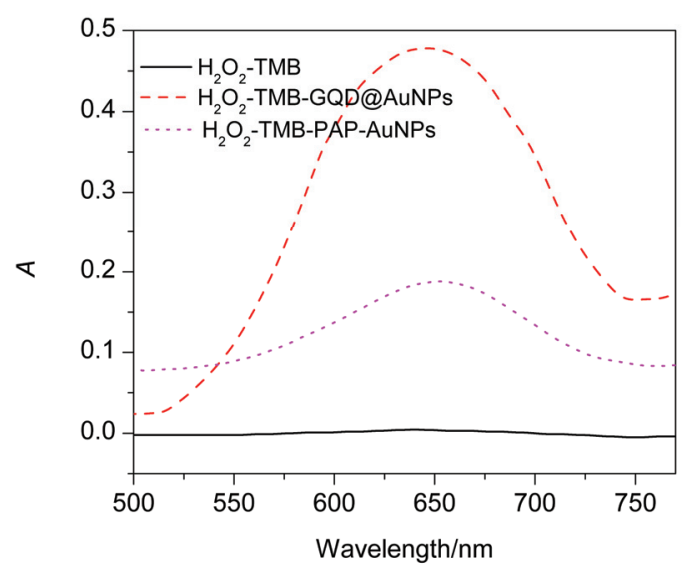

图 3 GQD@AuNPs, PVP-AuNPs 催化 $\mathrm{H}_{2} \mathrm{O}_{2}$ 氧化 TMB 的紫外吸收谱 图

Figure 3 The absorption spectra of GQD@AuNPs and PVP-AuNPs catalyzed $\mathrm{H}_{2} \mathrm{O}_{2}$ and TMB

为了体现 GQD $@ A u N P s$ 的优越性, 我们对比了相 关纳米材料的过氧化物酶催化活性(图 3), 合成了与 GQD@AuNPs 浓度、粒径基本一致的聚乙烯吡咯烷酮 (PVP)稳定的金纳米粒子(PVP-AuNPs). 可以看出, 相对 于 PVP 作保护剂制备的金纳米粒子, 用 GQD 作为保护 剂制备的金纳米粒子对 $\mathrm{H}_{2} \mathrm{O}_{2}$ 氧化 TMB 有更高的催化活 性. 这是由于 GQD 具有相对较好的导电性.

\section{4 影响 GQD@AuNPs 酶活性的因素}

与 HRP 类似, GQD@AuNPs 的催化活性依赖于体系 $\mathrm{pH}$ 值和温度. 分别考察了体系 $\mathrm{pH}$ 值和温度对 GQD@AuNPs 过氧化物模拟酶催化活性的影响, 结果 见图 4. 从图 $4 \mathrm{a}$ 可以看出, 在 $\mathrm{pH} 3.50$ 时吸光度最大, 这 
是因为 $\mathrm{pH}$ 小于 2 时易生成黄色的二亚胺物质, $\mathrm{pH}$ 大于 5 时过氧化氢分解加速, 因此在强酸性、中性的吸光度 均低于 $\mathrm{pH} 3.50$, 故我们选择优化的 $\mathrm{pH}$ 为 3.50. 图 $4 \mathrm{~b}$ 考察了反应温度从 $22 \sim 60{ }^{\circ} \mathrm{C}$ 升高时对 GQD@AuNPs 催化活性的影响, 结果表明 GQD@AuNPs 的最适温度 为 $40{ }^{\circ} \mathrm{C}$.
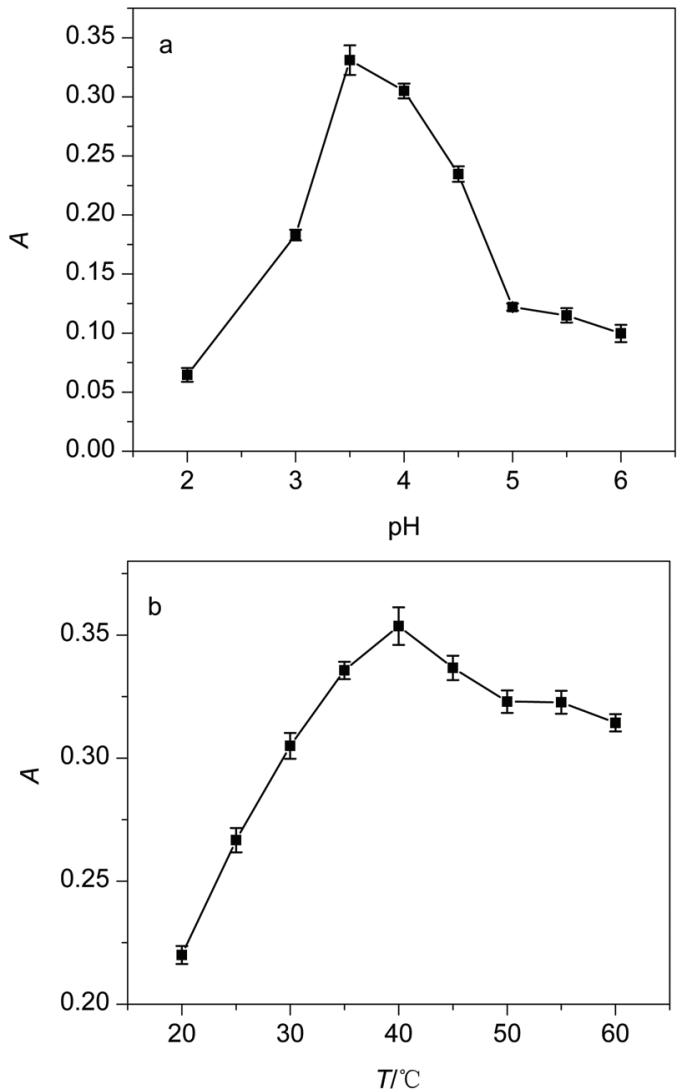

图 4 (a) $\mathrm{pH}$ 对 GQD@AuNPs 过氧化物酶活性的影响. 实验条件: 50 $\mu \mathrm{L}$ GQD@AuNPs, $1.00 \mathrm{mmol} \cdot \mathrm{L}^{-1} \mathrm{TMB}, 0.0300 \mathrm{mmol} \cdot \mathrm{L}^{-1} \mathrm{H}_{2} \mathrm{O}_{2}$, 反应 温度为 $40{ }^{\circ} \mathrm{C}$ 下 $10 \mathrm{~min}$. (b)温度对 GQD@AuNPs 过氧化物酶活性的影 响. 实验条件: $50.0 \mu \mathrm{L}$ GQD@AuNPs, $1.00 \mathrm{mmol} \cdot \mathrm{L}^{-1} \mathrm{TMB}, 0.0300$ $\mathrm{mmol} \cdot \mathrm{L}^{-1} \mathrm{H}_{2} \mathrm{O}_{2}, \mathrm{pH}$ 为 3.50

Figure 4 Effect of pH (a) and temperature (b) on peroxidase-like activity of GQD@AuNPs. Experiment condition: (a) $50.0 \mu \mathrm{L}$ GQD@AuNPs with $1.00 \mathrm{mmol} \cdot \mathrm{L}^{-1} \mathrm{TMB}$ and $0.0300 \mathrm{mmol} \cdot \mathrm{L}^{-1} \mathrm{H}_{2} \mathrm{O}_{2}$ as substrate at $40{ }^{\circ} \mathrm{C}$ for $10 \mathrm{~min}$; (b) $50.0 \mu \mathrm{L}$ GQD@AuNPs in HAc-NaAc buffer $(0.20$ $\mathrm{mol} \cdot \mathrm{L}^{-1}, \mathrm{pH} 3.50$ ) with $1.0 \mathrm{mmol} \cdot \mathrm{L}^{-1} \mathrm{TMB}$ and $0.0300 \mathrm{mmol} \cdot \mathrm{L}^{-1} \mathrm{H}_{2} \mathrm{O}_{2}$ as substrate

\section{5 稳态动力学及催化机理}

为了揭示 GQD@AuNPs 过氧化物模拟酶的催化反 应机理, 分别通过改变体系中 TMB 和 $\mathrm{H}_{2} \mathrm{O}_{2}$ 浓度测定了 显色反应的稳态动力学参数, 在特定的底物浓度范围 内, 酶催化反应遵循典型的 Michaelis-Menten 动力学模 式(图 5). 利用 Michaelis-Menten 双倒数方程 (Lineweaver-Burk plots): $1 / V=\left(K_{\mathrm{m}} / V_{\max }\left(1 /[\mathrm{S}]+1 / K_{\mathrm{m}}\right)\right.$ 法 可计算出酶催化反应的表观动力学常数: 最大初始反应 速率 $\left(V_{\max }\right)$ 和米氏常数 $\left(K_{\mathrm{m}}\right)$ (见表 1$)$. 由表 1 可知,
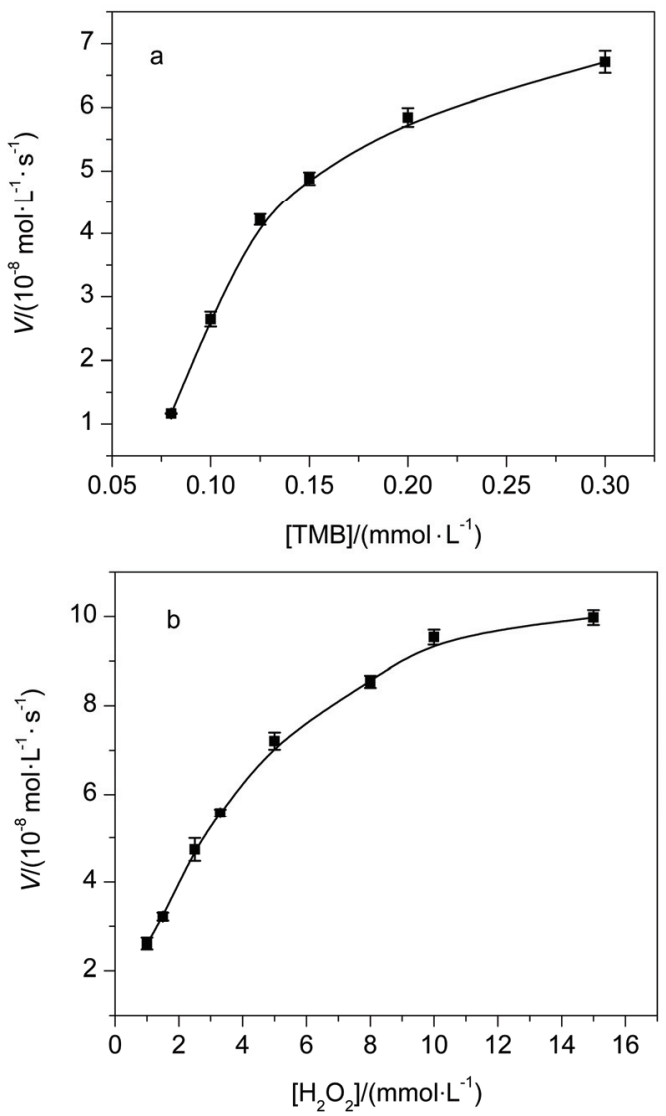

图 5 动力学分析: (a) 固定 $\mathrm{H}_{2} \mathrm{O}_{2}$ 浓度 $20 \mathrm{mmol} \cdot \mathrm{L}^{-1}$, 改变 $\mathrm{TMB}$ 浓度; (b) 固定 $\mathrm{TMB}$ 浓度为 $125 \mu \mathrm{mol} \cdot \mathrm{L}^{-1}$, 改变 $\mathrm{H}_{2} \mathrm{O}_{2}$ 浓度

Figure 5 Steady-state kinetic assay: (a) the concentration of $\mathrm{H}_{2} \mathrm{O}_{2}$ was $20 \mathrm{mmol} \cdot \mathrm{L}^{-1}$ and the TMB concentration was varied; (b) the concentration of TMB was $125 \mu \mathrm{mol} \cdot \mathrm{L}^{-1}$ and the $\mathrm{H}_{2} \mathrm{O}_{2}$ concentration was varied

表 $1 \mathrm{GQD} @ \mathrm{AuNP}$ 与 HRP 及其他不同纳米材料作为过氧化物模拟酶 的动力学参数比较

Table 1 Comparison of the kinetic parameters of the GQD@AuNP, HRP and other nanoparticles as peroxidase mimics

\begin{tabular}{|c|c|c|c|c|}
\hline Catalyst & Substance & $\begin{array}{c}K_{\mathrm{m}} / \\
\left(\mathrm{mmol} \cdot \mathrm{L}^{-1}\right)^{a}\end{array}$ & $\begin{array}{c}V_{\max } / \\
\left(10^{-8} \mathrm{~mol} \cdot \mathrm{L}^{-1} \cdot \mathrm{s}^{-1}\right)^{a}\end{array}$ & Reference \\
\hline \multirow[t]{2}{*}{ GQD@AuNP } & TMB & $0.033 \pm 0.029$ & $4.1 \pm 0.034$ & This work \\
\hline & $\mathrm{H}_{2} \mathrm{O}_{2}$ & $3.39 \pm 0.047$ & $7.48 \pm 0.041$ & \\
\hline \multirow[t]{2}{*}{ HRP } & TMB & 0.434 & 10.00 & [27] \\
\hline & $\mathrm{H}_{2} \mathrm{O}_{2}$ & 3.70 & 8.71 & \\
\hline \multirow[t]{2}{*}{ MWCNTs } & TMB & $0.077 \pm 0.032$ & $3.04 \pm 0.65$ & [27] \\
\hline & $\mathrm{H}_{2} \mathrm{O}_{2}$ & $33.1 \pm 5.6$ & $0.56 \pm 0.12$ & \\
\hline \multirow[t]{2}{*}{$\mathrm{Fe}_{3} \mathrm{O}_{4} \mathrm{NPs}$} & TMB & 0.098 & 3.44 & [28] \\
\hline & $\mathrm{H}_{2} \mathrm{O}_{2}$ & 154 & 9.78 & \\
\hline \multirow[t]{2}{*}{$\mathrm{Co}_{3} \mathrm{O}_{4} \mathrm{NPs}$} & TMB & 0.037 & 6.27 & [29] \\
\hline & $\mathrm{H}_{2} \mathrm{O}_{2}$ & 140.07 & 12.1 & \\
\hline \multirow[t]{2}{*}{ Carbon dots } & TMB & 0.039 & 3.61 & [30] \\
\hline & $\mathrm{H}_{2} \mathrm{O}_{2}$ & 26.77 & 30.61 & \\
\hline
\end{tabular}

GQD@AuNPs 对于两种底物 TMB 和 $\mathrm{H}_{2} \mathrm{O}_{2}$ 的 $K_{\mathrm{m}}$ 均低于 HRP. $K_{\mathrm{m}}$ 值越小, 说明酶对底物的亲和性越高. 这一结 
果表明 GQD@AuNPs 与 TMB 和 $\mathrm{H}_{2} \mathrm{O}_{2}$ 的亲和力比 HRP 与 TMB 和 $\mathrm{H}_{2} \mathrm{O}_{2}$ 的亲和力强. 而且 GQD@AuNPs 相对 于 TMB 的 $K_{\mathrm{m}}$ 小于一些常见的无机纳米材料如 $\mathrm{Fe}_{3} \mathrm{O}_{4}$, MWNT, $\mathrm{Co}_{3} \mathrm{O}_{4}$ 等, 说明 GQD $@ A u N P s$ 对 TMB 的亲和力 要高于这些纳米材料. 可能是因为 GQD 表面带有负电 荷, TMB 在酸性介质中以带有正电荷的分子状态存在, TMB 可以通过静电吸附在 GQD 表面, 因此 GQD@AuNPs 对 TMB 表现出较强的亲和力. 为了证实 这一推测, 测定了 $\mathrm{pH} 3.5$ 时 GQD 和 GQD@AuNPs 的 zeta 电位, 分别为 -27 和 $-13 \mathrm{mV}$, 说明 GQD 和 GQD@AuNPs 表面确实带有负电荷.

GQD@AuNPs 的催化特性从本质上应该与加速 $\mathrm{H}_{2} \mathrm{O}_{2}$ 分解产生 ${ }^{\circ} \mathrm{OH}$ 有关. 我们推测 GQD@AuNPs 可通 过与 $\mathrm{H}_{2} \mathrm{O}_{2}$ 反应使其 $\mathrm{O}-\mathrm{O}$ 键断裂而产生 ${ }^{\circ} \mathrm{OH}$, 生成的 'OH 在 GQD@AuNPs 表面氧化 TMB 使其显色. GQD@AuNPs 的过氧化物模拟酶催化活性优于单独的 GQD 和 AuNPs 的加和应该归因于协同作用. 当 GQD@AuNPs 加入到 TMB 和 $\mathrm{H}_{2} \mathrm{O}_{2}$ 的反应体系中, TMB 和 $\mathrm{H}_{2} \mathrm{O}_{2}$ 分别吸附在 GQD@AuNPs 表面, 由于 GQD 良 好的电子转移能力, $\mathrm{TMB}$ 上氨基的孤对电子转移给 GQD, 增加 GQD 费密能级和从 LUMO 到 AuNPs 的电 化学势, 使 GQD@AuNPs 表面的电子密度和流动性增 加, 进而加速了电子受体 $\mathrm{H}_{2} \mathrm{O}_{2}$ 与电子供体 GQD@AuNPs 之间的电子转移, 提高了 $\mathrm{H}_{2} \mathrm{O}_{2}$ 的分解能 力, 使一个过氧化氢分子加速形成 2 个 ${ }^{\circ} \mathrm{OH}$.

为了证明 GQD@AuNPs 的过氧化物模拟酶的催化 活性来自催化 $\mathrm{H}_{2} \mathrm{O}_{2}$ 分解产生的 ${ }^{\circ} \mathrm{OH}$, 借助于 ESR 光谱 对显色反应中产生的 ${ }^{\circ} \mathrm{OH}$ 进行表征(见图 6). 当加入 GQD@AuNPs 时, ESR 光谱出现 4 个典型的 DMPO/.OH 加合物的特征峰, 四条自旋共振谱线强度比为 $1: 2$ : $2 ： 1$, 这为 GQD@AuNPs 能催化过氧化氢分解产生 $\mathrm{OH}$ 提供了直接证据. 图 7 为 GQD@AuNPs 催化 TMB 和 $\mathrm{H}_{2} \mathrm{O}_{2}$ 反应可能的催化机理图.

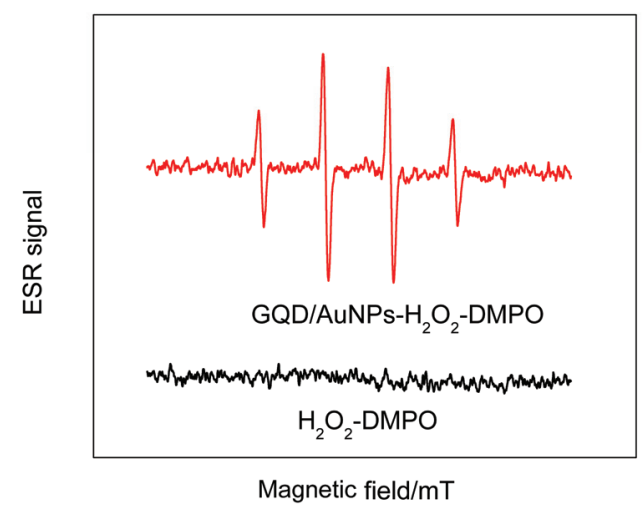

图 $6 \mathrm{GQD} @ \mathrm{AuNPs}$ 与 $\mathrm{H}_{2} \mathrm{O}_{2}$ 反应产生羟基自由基的 $\mathrm{ESR}$ 谱图

Figure 6 ESR spectra of hydroxyl radical generated via the reaction of DMPO probe in the GQD@AuNPs- $\mathrm{H}_{2} \mathrm{O}_{2}$ systems

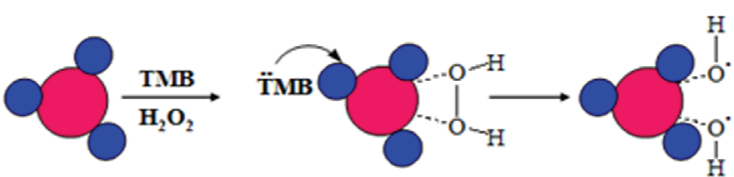

图 $7 \mathrm{GQD} @$ AuNPs 催化 TMB- $\mathrm{H}_{2} \mathrm{O}_{2}$ 反应可能的催化机理

Figure 7 Possible mechanism for the GQD@AuNPs-TMB- $\mathrm{H}_{2} \mathrm{O}_{2}$ system

\section{6 分析应用}

根据 GQD@AuNPs 能够催化 $\mathrm{H}_{2} \mathrm{O}_{2}$ 氧化 TMB 的酶 学性质, 结合 GOx 能够专一地酶催化葡萄糖产生 $\mathrm{H}_{2} \mathrm{O}_{2}$, 因此可以用 GQD@AuNPs 模拟酶代替传统的天然酶 $\mathrm{HRP}$ 间接比色测定葡萄糖. 图 8a 显示了不同浓度葡萄 糖在反应体系中所生成蓝色产物的颜色变化图. 在最佳 实验条件下, 葡萄糖的响应曲线和标准曲线如图 $8 \mathrm{~b}$ 所 示，在 $2.0 \times 10^{-6} \sim 4.0 \times 10^{-5} \mathrm{~mol} \cdot \mathrm{L}^{-1}$ 范围内葡萄糖浓度 与吸光度具有良好的线性关系 $(A=9.766 c+0.0116, r=$ $0.9983)$, 检出限为 $3.0 \times 10^{-7} \mathrm{~mol} \cdot \mathrm{L}^{-1}$. 该检出限比其他 一些纳米粒子做为过氧化物模拟酶的检出限低 $10 \sim 100$ 倍左右，见表 2. 本方法测定葡萄糖的灵敏度优于其他 纳米粒子这主要归因于 GQD 和 AuNPs 的协同耦合作用, 使得 GQD@AuNPs 的过氧化物模拟酶催化活性大大增 强. 因此, GQD@AuNPs 为构建一个简单、廉价、高灵 敏度的新型葡萄糖比色新方法提供了空间.
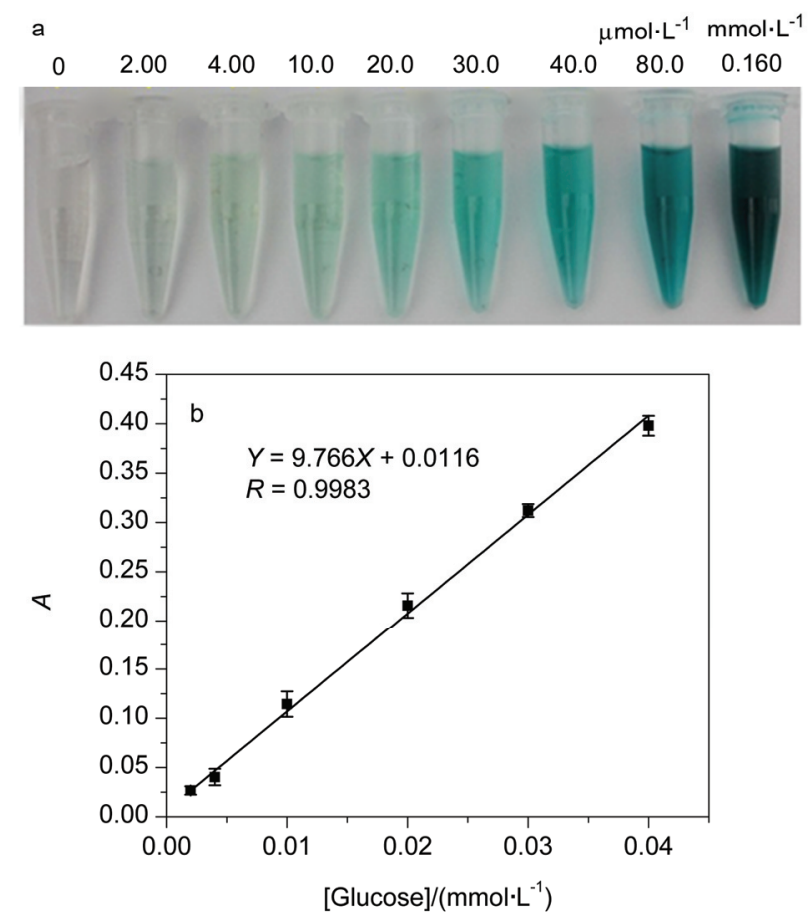

图 8 不同浓度葡萄糖生成蓝色产物的显色照片(a)和标准曲线(b) Figure 8 The photograph of production of colored products for different concentrations of glucose (a) and (b) the linear calibration plot for glucose

\section{7 方法选择性及生物样品测定}

由于酶催化的专一性，本方法对葡萄糖有很高的选 
表 2 不同纳米材料作为过氧化物模拟酶比色法检测葡萄糖的比较

Table 2 Comparison of colorimetric detection of glucose with different nanomaterials as peroxidase mimics

\begin{tabular}{cccccc}
\hline Catalyst & Substance Analytes & $\begin{array}{c}\text { Detection limit/ } \\
\left(\mu \mathrm{mol} \cdot \mathrm{L}^{-1}\right)\end{array}$ & $\begin{array}{c}\text { Linear range/ } \\
\left(\mu \mathrm{mol} \bullet \mathrm{L}^{-1}\right)\end{array}$ & Reference \\
\hline $\mathrm{Fe}_{3} \mathrm{O}_{4} \mathrm{NPs}$ & $\mathrm{TMB}$ & Glucose & 30 & $50 \sim 1000$ & {$[28]$} \\
Carbon NPs & TMB & Glucose & 20 & - & {$[31]$} \\
$\mathrm{Co}_{3} \mathrm{O}_{4} \mathrm{NPs}$ & $\mathrm{TMB}$ & Gucose & 5 & $10 \sim 1000$ & {$[29]$} \\
$\mathrm{MWCNTs}$ & $\mathrm{TMB}$ & Glucose & 8 & $8 \sim 100$ & {$[27]$} \\
$(+) \mathrm{AuNPs}$ & $\mathrm{TMB}$ & Glucose & 4 & $18 \sim 1100$ & {$[7]$} \\
$\mathrm{Co}_{3} \mathrm{O}_{4} / \mathrm{RGO}$ & $\mathrm{TMB}$ & Glucose & 1 & $1 \sim 100$ & {$[27]$} \\
$\mathrm{GQD}_{0} \mathrm{AuNP}$ & $\mathrm{TMB}$ & Glucose & 0.3 & $2 \sim 40$ & This work \\
\hline
\end{tabular}

择性. 图 9 比较了在相同条件下, 其他生物样品对本方 法的干扰. 相对于 $1 \mathrm{mmol} \cdot \mathrm{L}^{-1}$ 葡萄糖, $5 \mathrm{mmol} \cdot \mathrm{L}^{-1}$ 果糖、 $5 \mathrm{mmol} \cdot \mathrm{L}^{-1}$ 乳糖和 $5 \mathrm{mmol} \cdot \mathrm{L}^{-1}$ 麦芽糖均没有明显的检 测信号.

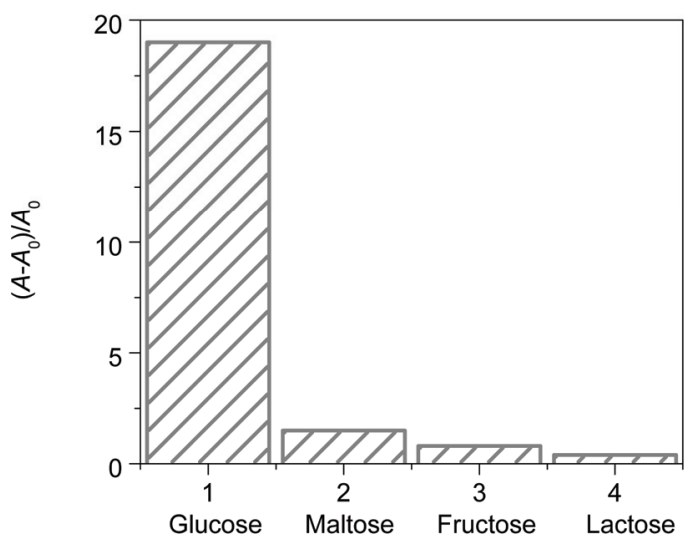

图 9 相对吸光度检测葡萄糖的选择性实验 (从左到右: $1 \mathrm{mmol} \cdot \mathrm{L}^{-1}$ 葡 萄糖, $5 \mathrm{mmol} \cdot \mathrm{L}^{-1}$ 麦芽糖, $5 \mathrm{mmol} \cdot \mathrm{L}^{-1}$ 果糖和 $5 \mathrm{mmol} \cdot \mathrm{L}^{-1}$ 乳糖)

Figure 9 Selectivity analysis for glucose detection by monitoring the relative absorbance (from left to right: glucose, maltose, fructose and lactose)

用所建立的方法对人体血清样品中葡萄糖的含量 进行了检测, 结果列于表 3 , 以样本所提供医院的测定 结果作为标准值 $(\mu)$, 置信度 $\mathrm{P}=90 \%$, 经 $\mathrm{t}$ 检验, 测定结 果与标准值之间不存在显著性差异, 即该方法能够成功 应用于实际样品中葡萄糖的检测分析.

表 3 人血清中葡萄糖的测定

Table 3 Determination of glucose in human serum sample

\begin{tabular}{lcccc}
\hline Sample & Official method ${ }^{a} /\left(\mathrm{mmol}^{\circ} \mathrm{L}^{-1}\right)$ & $\begin{array}{c}\text { This method }^{b} / \\
\left(\mathrm{mmol} \cdot \mathrm{L}^{-1}\right) \pm \mathrm{SD}\end{array}$ & $t$ & $t_{0.10,2}$ \\
\hline Serum 1 & 3.93 & $3.81 \pm 0.13$ & 1.73 & 2.92 \\
Serum 2 & 5.26 & $5.41 \pm 0.17$ & 1.52 & 2.92 \\
Serum 3 & 4.90 & $4.58 \pm 0.22$ & 2.51 & 2.92 \\
\hline
\end{tabular}

${ }^{a}$ Value from the Hospital of Shaanxi Normal University; ${ }^{b}$ This method mean \pm S.D $(n=3)$

\section{3 结论}

本文报道了用 GQD 既做保护剂又做还原剂, 在较 低温度下一步还原 $\mathrm{HAuCl}_{4}$ 的方法, 首次制备了 GQD@AuNPs. 得到的纳米粒子粒径小, 而且非常稳定. 此外，所制备的 GQD@AuNPs 具有优良的过氧化物模 拟酶的催化活性, 能够快速催化 $\mathrm{H}_{2} \mathrm{O}_{2}$ 氧化 TMB 显色, 其催化机理遵循 Michaelis-Menten 动力学模型. 通过 ESR 光谱为 GQD@AuNPs 催化反应体系中 ${ }^{\circ} \mathrm{OH}$ 产生提 供了直接证据. 最后, 利用 GQD@AuNPs 的酶催化活性 建立了一种可视化检测葡萄糖的新方法. 该法已成功用 于血样中葡萄糖的测定. 此方法不仅制备简单而且灵敏 度和选择性高. 本工作将有助于 GQD@AuNPs 过氧化 物模拟酶在环境化学、生物技术等领域的应用.

\section{4 实验部分}

\section{1 主要试剂与实验仪器}

葡萄糖、葡萄糖氧化酶、 $\mathrm{TMB}$ 购于上海阿拉丁试 剂有限公司; 辣根过氧化物酶购于 Sigma-Aldrich 公司; $\mathrm{HAuCl}_{4}$ (上海国药集团化学试剂有限公司). 实验用水为 超纯水(Millipore $18.2 \mathrm{M} \Omega \bullet \mathrm{cm}$ ). 血清样品来自陕西师范 大学校医院.

样品的形貌与尺寸观察测试采用 JEM-2100 TEM, (日本电子公司); 紫外-可见光谱实验在 U-3900H 紫外可见分光光度计(日本 Hitachi 公司)上进行; 光电子能谱 资料在 XPS (Kratos Analytical Ltd.)上获得.

\section{$4.2 \mathrm{GQD}$ 的制备}

GQD 的制备包括以下步骤：(1)氧化石墨烯的制 备 ${ }^{[32]}$. 称取 $2.5 \mathrm{~g} \mathrm{~K}_{2} \mathrm{~S}_{2} \mathrm{O}_{8}$ 和 $2.5 \mathrm{~g} \mathrm{P}_{2} \mathrm{O}_{5}$ 加入到 $12 \mathrm{~mL}$ 浓 $\mathrm{H}_{2} \mathrm{SO}_{4}$ 中, 加热溶解后, 再加入 $3.0 \mathrm{~g}$ 石墨粉, $80{ }^{\circ} \mathrm{C}$ 下反 应 $4.5 \mathrm{~h}$. 冷却到室温后用 $500 \mathrm{~mL}$ 超纯水稀释, 然后抽 滤洗涤，除去残留的酸和杂质离子，室温下干燥得到预 氧化的石墨. 将预氧化的石墨加入到 $120 \mathrm{~mL}$ 浓 $\mathrm{H}_{2} \mathrm{SO}_{4}$ 中, 冰浴下缓慢加入 $15 \mathrm{~g} \mathrm{KMnO}_{4}$, 并不断搅拌, 待加完 $\mathrm{KMnO}_{4}$ 后, 将体系升温至 $35{ }^{\circ} \mathrm{C}$ 反应 $2 \mathrm{~h}$. 然后加入超纯 水 $250 \mathrm{~mL}$, 搅拌 $2 \mathrm{~h}$, 再加入 $500 \mathrm{~mL}$ 超纯水和 $20 \mathrm{~mL}$ $30 \% \mathrm{H}_{2} \mathrm{O}_{2}$, 此时反应体系变为亮黄色. 将产物过滤, 用 1：10 的稀盐酸冲洗产物后再用大量水洗涤到中性，最 后将产物分散在超纯水中, 用分子量为 3500 的透析袋 透析并冻干后得到棕黄色氧化石墨烯粉末. (2) GQD 的 制备. 以氧化石墨烯为碳源, 利用超声一水热法制备了 $\mathrm{GQD}^{[33]}$. 具体步骤如下, 将 $23.3 \mathrm{mg}$ 氧化石墨烯分散在 $12 \mathrm{~mL}$ 超纯水中, 超声 $4 \mathrm{~h}$, 用 $\mathrm{NaOH}$ 调节 $\mathrm{pH}$ 为 8 , 将溶 液转移至聚四氟乙烯高压釜中, $200{ }^{\circ} \mathrm{C}$ 反应 $10 \mathrm{~h}$. 冷却 到室温后, 在 $15000 \mathrm{r} / \mathrm{min}$ 转速下离心 $30 \mathrm{~min}$, 将得到的 上层清液透析分离, 即得到 GQD 的水分散液. 


\subsection{GQD@AuNPs 的制备}

于烧瓶中加入 GQD $10.0 \mathrm{~mL}\left(0.20 \mathrm{mg} \cdot \mathrm{mL}^{-1}\right)$ 超声 $20 \mathrm{~min}$ 后, 置于磁力搅拌器上于 $100{ }^{\circ} \mathrm{C}$ 下加热回流 10 $\min$. 将 $500 \mu \mathrm{L}(0.5 \%)$ 的 $\mathrm{HAuCl}_{4}$ 滴加到热的 GQD 分散 液中, 继续加热搅拌 $50 \mathrm{~min}$, 溶液颜色变为酒红色, 离 心除去未反应的 GQD. 所获得的 GQD@AuNPs 重新分 散在超纯水中于 $4{ }^{\circ} \mathrm{C}$ 保存.

\section{4 葡萄糖的测定}

葡萄糖的测定分两步: (1)将 $20.0 \mu \mathrm{L}\left(5.0 \mathrm{mg} \bullet \mathrm{mL}^{-1}\right)$ 葡萄糖氧化酶 $(\mathrm{GOx})$ 和 $100.0 \mu \mathrm{L}$ 不同浓度的葡萄糖标 准液加入到 $200.0 \mu \mathrm{L}$ PBS 缓冲溶液 $(\mathrm{pH} 7.00,20 \mathrm{mmol} \cdot$ $\mathrm{L}^{-1}$ )中, $37{ }^{\circ} \mathrm{C}$ 水浴 $30 \mathrm{~min}$. (2)待冷却之后加入 $200.0 \mu \mathrm{L}$ TMB (2.50 mmol• $\left.\mathrm{L}^{-1}\right) 、 50.0 \mu \mathrm{L}$ GQD@AuNPs 和 630.0 $\mu \mathrm{L} \mathrm{HAc}-\mathrm{NaAc}$ 缓冲溶液 $\left(\mathrm{pH} 3.50,0.20 \mathrm{~mol} \cdot \mathrm{L}^{-1}\right.$ ), 将混合 溶液在 $40{ }^{\circ} \mathrm{C}$ 下孵育 $10 \mathrm{~min}$ 后, 于 $652 \mathrm{~nm}$ 处测其吸光 度. 血清中葡萄糖的测定首先用 $5000 \mathrm{Da}$ Amicon Cell 将 样品于 $8000 \mathrm{r} / \mathrm{min}$ 下超滤 $30 \mathrm{~min}$. 滤液稀释 10 倍, 以稀 释后的血清溶液代替上述葡萄糖标准溶液进行实验.

\section{References}

[1] Maxwell, D. J.; Taylor, J. R.; Nie, S. J. Am. Chem. Soc. 2002, 124, 9606.

[2] Zhang, H.; Cui, H. Nanoscale 2014, 6, 2563.

[3] Zhang, Q.; Wu, S.-Y.; He, M.-W.; Zhang, L.; Liu, Y.; Li, J.-H.; Song, X.-M. Acta Chim. Sinica 2012, 70, 2213. (张谦，吴抒遥，何 茂伟, 张玲, 刘洋, 李景虹, 宋溪明, 化学学报, 2012, 70, 2213.)

[4] Wei, H.; Wang, E. Chem. Soc. Rev. 2013, 42, 6060.

[5] Wallace, W. T.; Whetten, R. L. J. Am. Chem. Soc. 2002, 124, 7499.

[6] Chen, X.-H.; Du, J.-X. Acta Chim. Sinica 2011, 69, 745. (陈晓惠, 杜建修, 化学学报, 2011, 69, 745.)

[7] Jv, Y.; Li, B.; Cao, R. Chem. Commun. 2010, 46, 8017.

[8] Wang, S.; Chen, W.; Liu, A. L.; Hong, L.; Deng, H. H.; Lin, X. H. ChemPhys Chem 2012, 13, 1199.

[9] Wang, X.; Long, Y.; Wang, Q.; Zhang, H.; Huang, X.; Zhu, R.; Teng, P.; Liang, L.; Zheng, H. Carbon 2013, 64, 499.

[10] Du, L.; Huang, S.; Zhang, Q.; Jia, H.; Rockenbauer, A.; Liu, Y.; Liu,
K. J.; Liu, Y. Nanoscale 2014, 6, 1646.

[11] Tang, J.; Wang, J.; Wang, Y.; Li, Y.-F. Chin. J. Anal. Chem. 2011, 39, 1629. (唐杰, 王健, 王焱, 李原芳, 分析化学, 2011, 39, 1629.)

[12] Yin, H.-Z.; Liu, H.; Li, Y.-Y.; He, X.-W.; Chen, L.-X.; Li, W.-Y. Acta Chim. Sinica 2005, 63, 734. (尹洪宗, 刘辉, 李园园, 何锡文, 陈朗星, 李文友, 化学学报, 2005, 63, 734.)

[13] Rao, C. N. R.; Kulkarni, G. U.; Thomas, P. J. Chem. Soc. Rev. 2000 29, 27.

[14] Sun, X.-P.; Huang, M.-H.; Dong, S.-J.; Wang, E.-K. J. Fudan Univ. 2004, 43, 477. (孙旭平, 黄明华, 董绍俊, 汪尔康, 复旦学报, 2004, 43, 477.)

[15] Liu, W.; Yan, X.; Chen, J.; Feng, Y.; Xue, Q. Nanoscale 2013, 5, 6503.

[16] Zhao, J.; Chen, G.; Zhu, L.; Li, G. Electrochem. Commun. 2011, 13, 31.

[17] Li, L.; Wu, G.; Yang, G.; Peng, J.; Zhao, J.; Zhu, J. J. Nanoscale 2013, $5,4015$.

[18] Pan, D.; Guo, L.; Zhang, J.; Xi, C.; Xue, Q.; Huang, H.; Li, J.; Zhang, Z.; Yu, W.; Chen, Z.; Li, Z.; Wu, M. J. Mater. Chem. 2012, 22,3314 .

[19] Xie, W.-Q.; Fu, Y.-Y.; Ma, H.; Zhang, M.; Fan, L.-Z. Acta Chim. Sinica 2012, 70, 2169. (谢文箐, 傅英懿, 马红, 张沫, 范楼珍, 化 学学报, 2012, 70, 2169.)

[20] Ran, X.; Sun, H.; Pu, F.; Ren, J.; Qu, X. Chem. Commun. 2013, 49, 1079.

[21] Zhao, H.; Chang, Y.; Liu, M.; Gao, S.; Yu, H.; Quan, X. Chem. Commun. 2013, 49, 234.

[22] Habib, R.; Rahim, M. R. Biosens. Bioelectron. 2013, 41, 498.

[23] Alvarez, M. M.; Khour, J. T.; Schaaff, T. G.; Shafigullin, M. N.; Vezmar, W. R. L. J. Phys. Chem. B 1997, 101, 3706.

[24] Jaramillo, T. F.; Baeck, S. H.; Cuenya, B. R.; Mcfarland, E. W. J. Am. Chem. Soc. 2003, 125, 7148.

[25] Zhang, N.; Qiu, H.; Wang, W.; Li, Y.; Wang, X.; Gao, J. J. Mater. Chem. 2011, 21, 11080

[26] Pasricha, R.; Gupta, S.; Srivastava, K. Small 2009, 5, 2253

[27] Xie, J.-X. Ph.D. Dissertation, Southwest University, Chongqing, 2012. (谢建新, 博士论文, 西南大学, 重庆, 2012.)

[28] Gao, L. Z.; Zhuang, J.; Nie, L.; Zhang, J. B.; Zhang, Y.; Gu, N.; Wang, T. H.; Feng, J.; Yang, D. L.; Perrett, S.; Yan, X. Nat. Nanotechnol. 2007, 2, 577.

[29] Shi, W.; Zhang, X.; He, S.; Huang, Y. Chem. Commun. 2011, 47, 10785 .

[30] Shi, W.; Wang, Q.; Long, Y.; Cheng, Z.; Chen, S.; Zheng, H.; Huang, Y. Chem. Commun. 2011, 47, 6695.

[31] Wang, X.; Qu, K.; Xu, B.; Ren, J.; Qu, X. Nano Res. 2011, 4, 908.

[32] Xu, Y.; Bai, H.; Lu, G.; Li, C.; Shi, G. J. Am. Chem. Soc. 2008, 130, 5856.

[33] Sun, Y.; Long, H.; Dai, Y.; Jing, Y.; Zhang, L.; He, Y. $C N$ 102633257, 2012 [Chem. Abstr. 2012, 157, 389782]. 